\title{
Manejo de la hipertensión con biofeedback en la carga del cuidador: seguimiento y control de la hipertensión
}

\section{Management of hypertension with biofeedback in caregiver burden: monitoring and control of hypertension}

\author{
Antonio Aguilar-Agudo ${ }^{1,2 *}$ \\ ${ }^{1}$ Asociación Parkinson Córdoba; ${ }^{2}$ Grupo de investigación Comportamiento de riesgo, salud y seguridad laboral, Departamento de Psicología, \\ Facultad de Ciencias de la Educación, Universidad de Córdoba. Córdoba, España
}

Se presenta el caso de una mujer de 59 años que cuidaba de su esposo con enfermedad de Parkinson y demencia, que acudió por problemas relacionados con la carga del cuidador. Presentaba síntomas de estrés psicosocial, cefaleas, insomnio y tensión arterial (TA) alta. Había incrementado el consumo de tabaco, hasta ahora moderado. Fue derivada para valoración médica, consiguiendo normalizar la TA basal (Fig. 1) tras recibir tratamiento farmacológico (ramipril, $5 \mathrm{mg}, 1$ comp/día) para la hipertensión (HTA). Sin embargo, aumentos en la TA seguían siendo habituales en escenarios vividos como estresantes durante los cuidados hacia su cónyuge. Esto ponía en riesgo la salud de la paciente, siendo un obstáculo para continuar desempeñando el rol de cuidadora.

El Joint National Committee $7^{1}$ (JNC-7) propone adoptar estilos de vida saludables para el control de la HTA. Para el JNC-8², las decisiones sobre la atención deben considerar e incorporar las características clínicas y las circunstancias de cada paciente individual, y refleja que los médicos quieren orientación sobre el manejo de la HTA con la mejor evidencia científica posible. Las pautas del Colegio Americano de Cardiología y la Asociación Estadounidense del Corazón ${ }^{3}$ establecen la relación entre HTA y estrés psicosocial, situándolo como un factor de riesgo (FR) relativamente fijo. Varias publicaciones hacen referencia al efecto de las intervenciones no farmacológicas, mediante el uso de técnicas de meditación trascendental, sobre la TA, destacándose las debilidades metodológicas de este tipo de tratamientos ${ }^{4}$. Las pautas de 2017 enfatizan como objetivo para el tratamiento una presión sistólica $<130 \mathrm{mmHg}$ y diastólica $>80 \mathrm{mmHg}$.

En este sentido, el presente documento describe un tratamiento no farmacológico para el manejo de la HTA (no asociada a problemas orgánicos) y con ello la eliminación de un FR de enfermedades cardiovasculares (CV). El enfoque se centra en la estrategia de «cuidar al cuidador». La pregunta central al evaluar este método es determinar cómo el estudio llegó a sus conclusiones, siendo el propósito facilitar la comunicación clara de los hallazgos de la investigación para estimular la publicación de investigaciones relevantes.

Numerosas publicaciones muestran evidencias para considerar el tratamiento con biofeedback (BFB) como una alternativa que incrementa la conciencia de llevar un control sobre la TA, fomentando la adopción de un estilo de vida saludable ${ }^{5}$. Además, ofrece datos objetivos y medibles. La Guía ESC $2016^{6}$ propone como estrategia para facilitar el cambio conductual ayudar a 


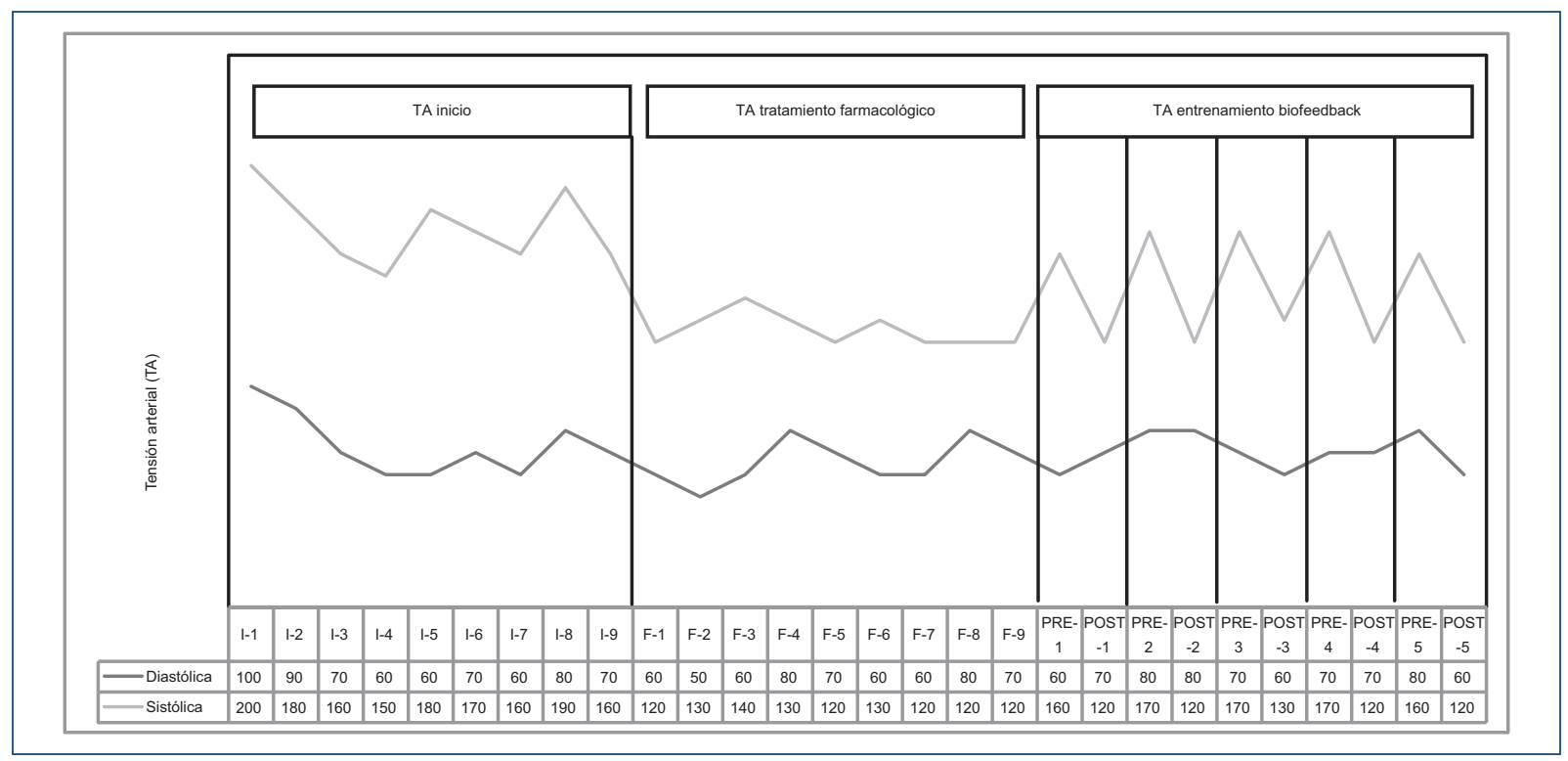

Figura 1. Registros de TA: al inicio (I); tras tratamiento farmacológico (F); durante cinco situaciones estresantes reales con medidas de TA antes (PRE) y después (POST) de realizar RA en casa.

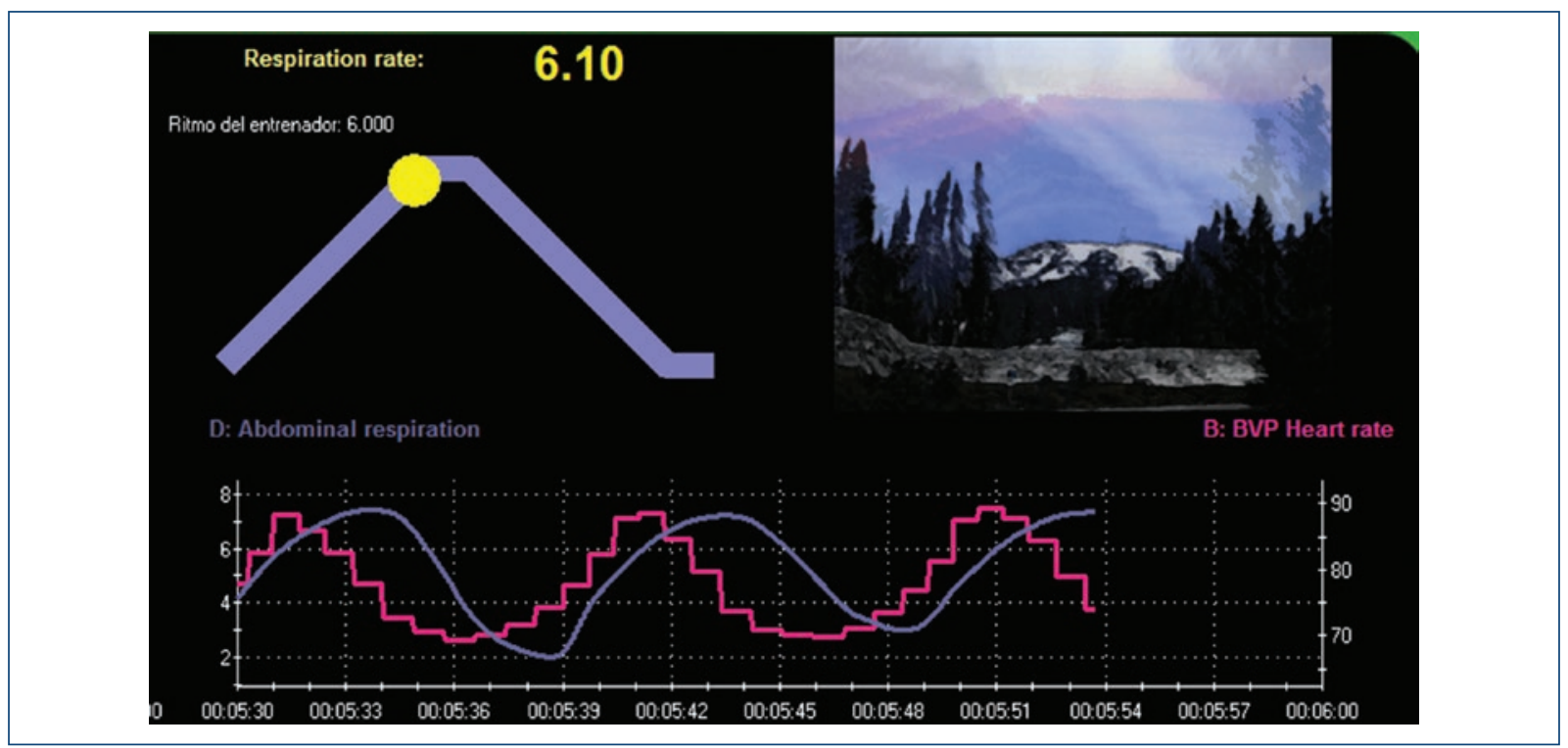

Figura 2. Señales visuales (RESP y BVP) proporcionadas a la paciente durante la fase de intervención (arriba el entrenador para los ejercicios de RA).

las personas a entender la relación entre su comportamiento y la salud. Los Comentarios a la Guía incluyen aportaciones acerca de la intervención individual sobre los FR: se destacan como estrategias hacer partícipe al paciente de su enfermedad y la recomendación de intervenir si el FR psicosocial es diagnosticable o puede contribuir a un empeoramiento de las enfermedades CV.
Durante el estudio, el entrenamiento con BFB se llevó a cabo utilizando el sistema ProComp5TM-Infini$\mathrm{ti}^{\circledR}$ (Thought Technology Ltd.), empleando los sensores BVP (volumen del pulso) y RESP (tasa respiratoria, TR). Durante cada sesión se proporcionaban señales visuales en una pantalla de ordenador: RESP, que podía medir frecuencia y amplitud abdominal en cada respiración, y en combinación con el BVP 
proporciona el seguimiento de la variabilidad de la frecuencia cardíaca (VFC). Se utilizó un protocolo para el entrenamiento de 6 respiraciones/minuto, como patrón para confluir en una VFC óptima. Durante cinco semanas se realizaron diez sesiones de veinte minutos. Se propuso la práctica en casa de los ejercicios de respiración abdominal (RA). Acabada la fase de entrenamiento, la paciente tenía habilidades para sincronizar su FC con la TR (Fig. 2) y había adquirido competencias para la ejecución de una técnica práctica y sencilla, sin necesidad de recibir las señales visuales.

Se hicieron algunos ensayos con la paciente, y se propuso su uso tras percibir ésta indicios de tensión y ansiedad en situaciones estresantes cotidianas. La revisión de los registros de TA (Fig. 1) indicaron una mayor habilidad en la paciente para mostrar un comportamiento relajado en situaciones estresantes tras la RA, provocando con ello una reducción de la TA. Durante todo el proceso se indicó a la paciente que el tratamiento farmacológico no debía ser interrumpido, correspondiendo cualquier decisión al respecto a su médico de referencia, que debía supervisar de manera habitual el control de la TA.

Este estudio describe cómo una paciente adquirió habilidades (patrón de respiración aprendido) para el manejo de TA alta asociada a los cuidados de su cónyuge. La reducción de la sintomatología que provoca la carga del cuidador mediante el desarrollo de habilidades para su autorregulación puede tener un impacto en la calidad de vida de los cuidadores y, como consecuencia, también en las personas receptoras de los cuidados.

\section{Financiamiento}

La presente investigación no ha recibido ayudas específicas provenientes de agencias del sector público, sector comercial o entidades sin ánimo de lucro.

\section{Conflicto de intereses}

El autor declara no tener conflicto de intereses.

\section{Responsabilidades éticas}

Protección de personas y animales. Los autores declaran que los procedimientos seguidos se conformaron a las normas éticas del comité de experimentación humana responsable y de acuerdo con la Asociación Médica Mundial y la Declaración de Helsinki.

Confidencialidad de los datos. Los autores declaran que han seguido los protocolos de su centro de trabajo sobre la publicación de datos de pacientes.

Derecho a la privacidad y consentimiento informado. Los autores han obtenido el consentimiento informado de los pacientes y/o sujetos referidos en el artículo. Este documento obra en poder del autor de correspondencia.

\section{Bibliografía}

1. Chobanian AV, Bakris GL, Black HR, Cushman WC, Green LA, Izzo JL Jr, et al.; Joint National Committee on Prevention, Detection, Evaluation, and Treatment of High Blood Pressure. National Heart, Lung, and Blood Institute; National High Blood Pressure Education Program Coordinating Committee. Seventh Report of the Joint National Committee on Prevention, Detection, Evaluation, and Treatment of High Blood Pressure. Hypertens. 2003;42:1206-52.

2. James PA, Oparil S, Carter BL, Cushman WC, Dennison-Himmelfarb C Handler J, et al. 2014 Evidence-based guideline for the management of high blood pressure in adults: Report from the Panel Members Appointed to the Eighth Joint National Committee (JNC 8). JAMA. 2014;311(5):507-20.

3. Whelton PK, Carey RM, Aronow WS, Casey DE Jr, Collins KJ, Dennison Himmelfarb C, et al. 2017 ACC/AHA/AAPA/ABC/ACPM/AGS/APhA/ASH/ ASPC/NMA/PCNA Guideline for the Prevention, Detection, Evaluation, and Management of High Blood Pressure in Adults: Executive Summary: A Report of the American College of Cardiology/American Heart Association Task Force on Clinical Practice Guidelines. Hypertens. 2018;71:1269-324.

4. Canter PH, Ernst E. Insufficient evidence to conclude whether or no Transcendental Meditation decreases blood pressure: results of a systematic review of randomized clinical trials. J Hypertens. 2004;22(11):2049-54.

5. Nakao M, Yano E, Nomura S, Kuboki T. Blood pressure-lowering effects of biofeedback treatment in hypertension: a meta-analysis of randomized controlled trials. Hypertens Res. 2003;26:37-46.

6. Arrarte V, Berenguel A, Gonzalez C, de la Hera JM, Lekuona I, Marzal D, et al. Guía ESC-2016 sobre prevención de la enfermedad cardiovascular en la práctica clínica. Rev Esp Cardiol. 2016;69(10):939.

7. Arrarte V, Berenguel A, Gonzalez C, de la Hera JM, Lekuona I, Marzal D, et al. Comentarios a la guía ESC-2016 sobre prevención de la enfermedad cardiovascular en la práctica clínica. Rev Esp Cardiol. 2016;69(10):894-9. 